\title{
Chapter 70 \\ Resonant Optical Trapping \\ in Microfluidic-Integrated Hollow Photonic Crystal Cavities
}

\author{
Rita Therisod, Mario Tonin, and Romuald Houdré
}

\begin{abstract}
Optical trapping of nanoparticles has many interesting applications in a variety of physical and biological studies. The ability of photonic crystal cavities to strongly confine light permits to overcome the diffraction limit that prevents the trapping of submicron sized particles with low powers in classical optical tweezers. The device studied in this project is integrated in an optofluidic chip. Distinction between 250 and $500 \mathrm{~nm}$ particles is shown.
\end{abstract}

R. Therisod $(\varangle) \bullet M$. Tonin $\bullet$ R. Houdré

Laboratory of Advanced Semiconductors for Photonics and Electronics,

École Polytechnique Fédérale de Lausanne, Lausanne, Switzerland

e-mail: rita.therisod@epfl.ch 